\title{
The Wonder of Colors and the Principle of Ariadne
}

\author{
Walter Carnielli and Carlos di Prisco
}

\section{Why Colors Matter for Mathematics}

The aim of this paper is to survey, and motivate the foundational importance of, an infinitary principle that is independent of the Axiom of Choice in $\mathrm{ZF}$, albeit it can be consistently added to the remaining axioms. It concerns a principle which we call the Principle of Ariadne, formulated almost 30 years ago in the powerful language of colors in Carnielli and Di Prisco (1988) and later published as Carnielli and Di Prisco (1993).

Despite the importance of colors in cultural history and art, philosophers have doubts whether colors exist only in our mind. Nonetheless, colors are a subject of intense use, abuse and discussion: are colors just used as a sense of similarity, while we do not have any better notions to classify certain things? Quine seemed to think that colors are not natural kinds (Quine 1969) and have no significance in theoretical science: colors simply do not participate in laws of nature. But Wittgenstein was more emphatic and positive while trying to establish the logic of color concepts: "Colours are a stimulus to philosophizing", he remarked, in an attempt to explain Goethe's passion for the theory of colors: "Colours seem to present us with a riddle, a riddle that stimulates us, not one that exasperates us." (Wittgenstein 1998, p. 76, written in 1948).

\footnotetext{
W. Carnielli $(\triangle)$

Department of Philosophy, Centre for Logic, Epistemology and the History of Science, State University of Campinas UNICAMP, Campinas, SP, Brazil

e-mail: walter.carnielli@cle.unicamp.br; walter.carnielli@gmail.com

C. di Prisco

Instituto Venezolano de Investigaciones Científicas, Universidad de Los Andes, Bogotá, Colombia e-mail: cdprisc@ivic.gob.ve; cdiprisc@gmail.com
} 
Mathematicians, on the completely opposite side, are not concerned whether colors are natural kinds or not, and really see colors as a stimulus to mathematizing: colors are used in finite and infinite combinatorics as the best way to understand and think about mathematical similarities. From this vantage point, colors provide extraordinary help for the heuristics of mathematics, and literally hundreds of problems, several of them of great deepness and difficulty, are versed in the form of questions about colors and ways of coloring. Some of them touch the bounds of logic and foundations of set theory, as we shall see.

To appreciate the heuristic power of the language of colors in mathematics, the famous Four-Colors Theorem for planar graphs (maps) is a good example. This problem first appeared in 1852 in a letter from Augustus De Morgan to William Rowan Hamilton, only solved using computers in 1976 by Kenneth Appel and Wolfgang Haken. It states that any planar map is colorable with at most four colors.

A mathematical equivalent of this problem is the following: given a partition of a plane into contiguous regions, if an attribute is assigned to each region, no more than four attributes are required so that no two adjacent regions have the same attribute. Here, two regions are adjacent iff they share a common boundary. Formulated in such a dry language, it becomes obvious that without referring to maps and colors it is possible that nobody would had thought about such a problem.

One of the first, and more fundamental, principles that guide combinatorial arguments is the famous Schubfachprinzip, ("drawer principle" or "shelf principle") devised by Peter Gustav Lejeune Dirichlet in 1834, nowadays better known as Dirichlet's pigeonhole principle (DPP)

Principle 1 Given $n$ pigeons distributed in $m$ pigeonholes, if $n>m$ (i.e., the number of pigeons is strictly bigger than the number of pigeonholes) then at least one pigeonhole contains at least two pigeons.

DPP and can be easily re-stated in terms of colors:

Principle 2 Given $n$ objects painted with $m$ colors, if $n>m$ (i.e., the number of objects is strictly bigger than the number of colors) then there are at least two objects with the same color.

DPP seems obvious and can be proved by elementary means (a usual proof is by reductio ad absurdum), but it is really the basis for much deep generalization. Heuristically, changing the perspective from "pigeonholes" to "colors" permits us to extend the inherent intuition of DPP to any number, finite or infinite, of colors, infinite sets, hypergraphs, etc.

In 1930, while investigating properties of formal logic, Frank Ramsey proved a remarkable generalization of DPP in Ramsey (1930). By solving a special case of the Entscheidungsproblem of Hilbert and Ackermann, to wit, the decidability for validity of the class of the $\exists \forall$-sentences with identity, Ramsey originated the deeply studied area known as Ramsey theory. It is convenient first to briefly survey some finite cases of Ramsey structures more connected to graph theory. An undirected graph consists of a collection of vertices and a collection of edges (formally, 
unordered pairs of vertices). ${ }^{1}$ A graph is complete when it has an edge between every pair of vertices. A version of Ramsey's theorem can be stated as follows.

Theorem 3 Finite Ramsey's Theorem for pairs and 2 Colors. Given two colors (blue and red) and a pair $(p, q)$ of positive integers, there exists a minimal positive integer $R(p, q)$ such that any complete graph with $R(p, q)$ vertices whose edges are colored blue or red contains either an entirely blue complete subgraph with $p$ vertices or an entirely red complete subgraph with $q$ vertices.

A simple and illustrative case of the Finite Ramsey's Theorem for 2 colors is the Meeting Theorem, which explains why in any conference with at least six people there are always three mutual friends or three people that need to be formally introduced to each other:

Theorem 4 Meeting Theorem. In any meeting with 6 or more participants there is always (at least) 3 of them whom are either mutual strangers or mutual acquaintances.

The Meeting Theorem corresponds to the statement $R(3,3)=6$. The finite Ramsey number $R(m, n)$ is the smallest number of people who should be participating in a conference to guarantee that $m$ people all know each other, or $n$ people are mutual strangers.

Theorem 5 Generalized Finite Ramsey's Theorem. Given any number $k$ of colors and any $k$-tuple $\left(n_{1} \ldots, n_{k}\right)$ of positive integers, there exists a positive integer $R\left(n_{1} \ldots, n_{k}\right)$, known as the $\left\langle n_{1} \ldots, n_{k}\right\rangle$-Ramsey number, such that, if the edges of the complete graph with $R\left(n_{1} \ldots, n_{k}\right)$ vertices are colored with $k$ distinct colors, then there exists a complete monochromatic subgraph with $n_{i}$ vertices for some color $1 \leq i \leq k$.

The proof of the Generalized Finite Ramsey's Theorem is by induction. It is not substantially deeper than the Finite Ramsey's Theorem for 2 Colors, but computationally much harder. The proof gives constructive upper bounds, while a major computational issue is to find constructive lower bounds. Explicit computations of the values of the Ramsey numbers $R\left(n_{1}, \ldots, n_{c}\right)$ are extremely difficult, and only a few of them have been calculated. Even the exact values of $R(r, s)$ for small $\mathrm{r}$ and s are open: for instance, it is known that $43 \leq R(5,5) \leq 49$, and perhaps with the extra-computer power of this century the exact value could be computed. But mankind ${ }^{2}$ is not prepared for computing $R(6,6)$.

The metamathematical, if not philosophical, moral behind Ramsey Theory is that complete disorder is impossible: given enough resources, some degree of selforganization is inevitable. This aspect is discussed in Carnielli (1996).

\footnotetext{
${ }^{1}$ We're considering here only undirected graphs. A directed graph consists of a collection of vertices and a collections of arcs (ordered pairs of vertices). Finite Ramsey's Theorems also extend to directed graphs, but are slightly more complicated.

${ }^{2}$ Paul Erdôs is reported to have said: "If the demon asked us to tell him the value of $R(6,6)$ we should devote all our resources to finding a way to kill the demon". Erdős was well aware that it would be easier to kill a demon than to compute $R(6,6)$.
} 


\section{Colored Partition Relations}

The Generalized Finite Ramsey's Theorem is immediately extendable to infinite cardinals in several directions, including many colors.

Let $[\mathbb{N}]^{[n]}=\{a \subseteq N:|a|=n\}$ denote the set of all finite sets $a$ of natural numbers with cardinality $n$. A version of the deep result proved in Ramsey (1930) is the following:

Theorem 6 Infinite Ramsey's Theorem for $n$-subsets and $k$-colors $\left(R_{k}^{n}\right)$. Given positive integers $n$ and $k$, for every coloring of $[\mathbb{N}]^{[n]}$ in $k$ colors there exists an infinite subset of natural numbers $H \subset \mathbb{N}$ such that $[H]^{[n]}$ (the collection of all of all n-element subsets of H) is monochromatic. Such a set $H$ is said to be homogeneous for the coloring.

When only cardinality matters, P. Erdôs and R. Rado introduced in Erdôs and Rado (1956) the "arrow notation", which is more appropriate for set-theoretical investigations. Let $\kappa$ and $\lambda$ be cardinals. The abbreviation

$$
\kappa \rightarrow(\lambda)_{\mu}^{n}
$$

means that for every $\mu$-coloring of the set $[\kappa]^{n}$ of $n$-element subsets of $\kappa$ in $\mu$ colors there is a homogeneous set of size $\lambda$. When not specified, $\mu=2$.

In arrow notation, Ramsey's Infinite Theorem is usually written as $\omega \rightarrow(\omega)_{k}^{n}$, meaning that for all positive integers $n$ and $k$ and every $k$-coloring of all the $n$ element subsets of a denumerably infinite set $X$ contains all the $n$-element subsets of an infinite set $Y$.

Ramsey's Infinite Theorem for well-ordered sets (such as $\mathbb{N}$ ) does not need the Axiom of Choice. But the Axiom of Choice for countable families of finite sets is equivalent to Ramsey's infinitary statement for arbitrary sets (Lolli 1977). Standard proofs of this theorem are framed in the setting of the axioms of ZFC set theory, but such axioms may be more powerful than necessary. This point will be addressed below.

Ramsey theory provides an illuminating example of why the theory of finite sets does not coincide with Peano Arithmetic, as proved by Paris and Harrington in (1977): they found a finite variant of Ramsey's theorem expressible in first order arithmetic that is true in $\mathbb{N}$, unprovable in Peano Arithmetic, but provable in ZF set theory, as Gödel had anticipated in his incompleteness theorems.

In the 1950s Erdős and Rado (1956) extended Ramsey's result in several directions with their so-called partition calculus. The development of the theory of polarized partition relations, related to problems of partitions of sequences of subsets instead of just subsets, was further developed in Erdős et al. (1965), and partitions into any finite number of pieces and several results are studied in Di Prisco and Henle (1993) and Carrasco et al. (1995).

Partition properties are usually stated in terms of $k$ colorings. Again, because colorings establish similarities without substantial distinction, colors provide help with heuristics. 
A particularly interesting kind of partition relation problem is the following: given a coloring $F: \omega^{\omega} \mapsto 2$ of the collection $\omega^{\omega}$ of all infinite sequences of natural into two colors, is there a sequence $H_{0}, H_{1}, \cdots$ of subsets of $\omega$ such that $F$ is constant on $\Pi_{i \in \omega} H_{i}$ ? In other words, given a 2-coloring of all the infinite sequences of natural numbers, is there a monochromatic infinite Cartesian product $\Pi_{i \in \omega} H_{i}$ under $F$ ?

The answer obviously depends on the cardinality required for the $H_{i}$ s. If there is no restriction and $H_{i}$ 's can be taken to be singletons, the answer is clearly positive: any infinite sequence $<n_{1}, n_{2}, \cdots, n_{k}, \cdots$ of any color will do the trick: just take $H_{i}=\left\{n_{i}\right\}$ and then the product $\Pi_{i \in \omega} H_{i}$ is monochromatic as it has only one element. But surprises emerge when all $H_{i} \mathrm{~s}$, or infinitely many of them, are required to have at least two elements: requiring an infinite number of $H_{i}$ s with $\left|H_{i}\right| \geq 2$ dramatically changes the problem.

Let the symbol

$$
\left(\begin{array}{c}
\omega \\
\omega \\
\vdots
\end{array}\right) \rightarrow\left(\begin{array}{c}
2 \\
2 \\
\vdots
\end{array}\right)
$$

represent the validity of the following polarized partition relation:

$A_{\omega}$ : "for any coloring $F: \omega^{\omega} \mapsto 2$ there is a sequence $H_{0}, H_{1}, \cdots$ of subsets of $\omega$ with each $H_{i}$ s containing two or more elements such that $F$ is constant on $\Pi_{i \in \omega} H_{i}$ ",

The following result can be proved:

\section{Theorem 7}

$$
\left(\begin{array}{c}
\omega \\
\omega \\
\vdots
\end{array}\right) \rightarrow\left(\begin{array}{c}
2 \\
2 \\
\vdots
\end{array}\right)
$$

contradicts the Axiom of Choice.

A short proof of the contradiction of $A_{\omega}$ with the Axiom of Choice is given in Carnielli and Di Prisco (1993) where the topic is further developed.

However, the statement $A_{\omega}$ is relatively consistent with ZF under certain conditions. In more precise terms, $A_{\omega}$ is consistent with $\mathrm{ZF}$ plus the axiom of dependent choice $(\mathrm{ZF}+\mathrm{DC})$ assuming the consistency of Zermelo-Fraenkel set theory with the axiom of choice (ZFC) together with the existence of an inaccessible cardinal. This follows from a result of Mathias (1977) (see Carnielli and Di Prisco 1993 for a discussion). In this way, the statement $A_{\omega}$ can be regarded as a new principle to be added to the foundations of mathematics, with interesting consequences, some of them to be further explored, as we discuss below. 


\section{The Principle of Ariadne and the Foundations of Mathematics}

It is interesting that some results in Carnielli and Di Prisco (1993) were inspired by a variant of Ramsey's finite theorem for sequences introduced in Carnielli (1986). So a finitary principle that works well under ordinary induction, when expanded towards the transfinite may present some unexpected behavior.

In the Greek myth of the Minotaur, Ariadne gave Theseus a ball of thread, which he unrolled while descending into the labyrinth. This allowed him to find his way back out, despite the Minotaur. As an analogy to help to understand the interest of $A_{\omega}$, consider the following game: suppose that Daedalus, the skillful engineer has now built an infinite labyrinth, corresponding to $\omega^{\omega}$. To confuse the intruders, Daedalus has painted each infinite bottom-up way red or blue (that is, applied a 2coloring $F: \omega^{\omega} \mapsto 2$ ), and the design of the labyrinth guarantees that if someone choose a color and then follows a path from the bottom, he/she will escape from the labyrinth if at the end the path turns out to be of the chosen color.

The Ariadne game is defined by the following rules: Theseus has to choose a color and to follow a path painted with the chosen color. Notice that the color will only become visible when the journey along the infinite path is completed, as $F$ assigns colors to infinite sequences.

Each time Theseus moves from a point $n_{i}$ at level $i$ to a point $n_{i+1}$ at level $i+1$, the Minotaur goes right before him and cuts a path from level $i+1$ to $i+2$. The Minotaur wins if Theseus cannot escape, and Ariadne wins if Theseus gets back to her. How can Theseus escape the labyrinth? Instead of a ball of thread, Ariadne now tells Theseus a color $c$ and a secret sequence $H_{0}, H_{1}, \cdots$ such that all paths from the bottom in the infinite product $\Pi_{i \in \omega} H_{i}$ are monochromatic with color $c$. Even if the Minotaur will cut a path from move to move, there will always be an infinitely long path from the bottom leading to the way out, and Ariadne has a winning strategy. $A_{\omega}$ pictorially called the Principle of Ariadne, corresponding to a winning strategy to the Ariadne Game.

To see, intuitively, why Theseus can always escape, suppose for instance that Ariadne informed him that the product

$$
M=H_{0} \times H_{1} \times H_{2} \times H_{3} \times \cdots
$$

is blue, where $H_{0}=\{1,4\}, H_{1}=\{3,5\}, H_{2}=\{7,5\}, H_{3}\{3,6\}$.

This means that all paths $\langle 1,3,5,3, \cdots\rangle,\langle 4,5,5,6, \cdots\rangle$, etc., in the Cartesian product $M$ have the same color blue:

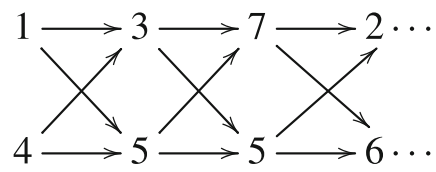


If Theseus (after choosing blue as his color) is at point 1 of level 0 and the Minotaur cuts the connection $\langle 1,5\rangle$, he then goes to 3 at level 1 . From 3, if the Minotaur cuts the connection $\langle 3,7\rangle$, Theseus goes to 5 at level 2 . And from there, if the connection $\langle 5,6\rangle$ is cut, he goes to 2 at level 3 , and so on.

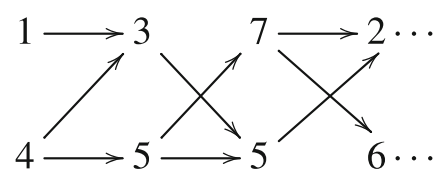

Thus, in any case, Theseus progresses uninterruptedly on a blue thread from the bottom and eventually escapes the labyrinth.

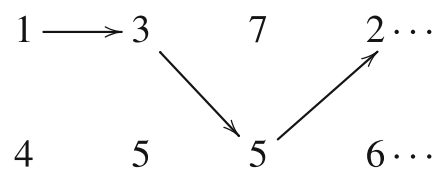

\section{On Alternative Set Theories and Mathematical Pluralism}

In the course of the development of contemporary foundations of mathematics a number of alternative axioms have been proposed defining the so-called alternative set theories, adding to Zermelo-Frankel set theory different principles for different purposes. It is well known that the axioms of $Z F C$, Zermelo-Fraenkel set theory with the axiom of choice, cannot settle all interesting questions of mathematics. The question posed by the Continuum Hypothesis is emblematic: work of Gödel and of Cohen show that the Continuum Hypothesis cannot be proved nor disproved from the axioms of $Z F C$. The search for new natural axioms that decide the value of the continuum has been one of the leading motivations for the development of the foundations of set theory. In the course of this search different new principles have been considered giving rise to a diversity of extensions of $Z F$ or $Z F C$. Other axioms have been proposed with different motivations and many of them have turn out to be extremely interesting and have uncovered un expected relations between different parts of mathematics. The Axiom of Determinacy is an example of particular interest (see Mycielski and Steinhaus 1962). It contradicts the axiom of choice, but together with weak choice principles provides a very interesting theory of sets of real numbers. The relation of the axiom of determinacy and large cardinals is a very deep and rich part of contemporary mathematics. The need for new axioms has been discussed amply during the recent decades (see, for example Feferman et al. 2000) 
The Principle of Ariadne, among others, would constitute an alternative to the familiar Axiom of Choice, whose interest is still to be investigated. A variety of related principles and their relation with $\mathrm{AC}$ has been considered, see for instance Di Prisco and Henle (1999).

It was proved in Di Prisco and Todorcevic (2003) that the classical partition relation $\omega \rightarrow(\omega)^{\omega}$ is not equivalent to its polarized version, solving a longstanding problem in the area. Moreover, the paper also shows that, although the Principle of Ariadne contradicts the Axiom of Choice, it is consistent with some of its consequences. The consistency of $\mathrm{ZF}+\mathrm{DC}+$ Principle of Ariadne + there exists a non-principal ultrafilter in $\omega$ is proved there, supposing the consistency of ZCF and the existence of an inaccessible cardinal.

A particularly interesting problem would be to investigate the relationship between the Principle of Ariadne and the Axiom of Determinacy mentioned above.

Theorem 7 shows that the Principle of Ariadne is hopeless in the presence of the Axiom of Choice: a 2-coloring for which there is no homogeneous infinite sequence $H_{i}, i \in \omega$ can always be produced using the Axiom of Choice. Polarized partition relations have also certain incompatibilities with the Generalized Continuum Hypothesis, as shown in Shelah (1998), where it is proved that for a strong limit singular cardinal $\lambda$ at which the generalized continuum hypothesis fails (i.e. $2^{\lambda}>\lambda^{+}$), a polarized partition relation holds.

A diametrically opposed relationship between the finite and the infinite was obtained in a recent surprising result of Patey and Yokoyama (2016) about the prooftheoretic strength of Ramsey's theorem $R_{2}^{2}$ for pairs and two colors. They proved that $R_{2}^{2}$ is finitistically reducible, in the sense of the reverse mathematics program and its realization of Hilbert's Program.

Such a result can be seen as a kind of bridge connecting the finite and the infinite, in the sense that any finitistic consequence of the infinite machinery of $R_{2}^{2}$ can be provable without access to infinity. In other words, infinite tools make the proof easier to find, but can always be replaced by another, perhaps more involved, finitary proof. But apparently these arguments only work for $R_{2}^{2}$ : even the next 2-colorable case $R_{2}^{3}$ reveals to be disconsolately far from any finitistic reasoning.

Alternative set theories represent the freedom of mathematical pluralism, the possibilities of constructing different mathematical worlds from the one we experience today. The consequences of the Principle of Ariadne are not fully investigated, but the fact that it can be added to the machinery of an alternative mathematics is certainly attractive for research, promising further bewilderment if we continue gazing on the face of the infinite.

Acknowledgements Some results in this paper have been written years ago during a visit of the first named author to the Instituto Venezolano de Investigaciones Científicas (IVIC) in Caracas, Venezuela. This author also acknowledges support from FAPESP Thematic Project LogCons 2010/51038-0, Brazil, and from a research grant from the National Council for Scientific and Technological Development (CNPq), Brazil. 


\section{References}

Carnielli, W. A. (1986). Ramsey-type theorems for cubes. In A. M. Gleason (Ed.), Abstracts of the International Congress of Mathematicians '86, Berkeley (p. 305). American Mathematical Society.

Carnielli, W. A. (1996). Auto-organização em estruturas combinatórias. In O. Pessoa, Jr., M. M. Debrun, \& M. E. Q. Gonzalez (Eds.), Auto-Organização: Estudos Interdisciplinares (Vol. 18, pp. XX-YY). Coleção CLE, CLE- Unicamp.

Carnielli, W. A. \& Di Prisco, C. A. (1988). Polarized partition relations for higher dimension (Vol. 12). Technical report, Department of Mathematics, University of Campinas. Published as Relatório Técnico do Instituto de Matemática da Universidade Estadual de Campinas.

Carnielli, W. A. \& Di Prisco, C. A. (1993) Some results on polarized partition relations of higher dimension. Mathematical Logic Quarterly, 39, 461-474.

Carrasco, M., Di Prisco, C. A., \& Millán, A. (1995). Partitions of the set of finite sequences. Journal of Combinatorial Theory, Series A, 71(2), 255-274.

Erdős, P. \& Rado, R. (1956). A partition calculus in set theory. Bulletin of the American Mathematical Society, 62(5), 427-489.

Erdős, P., Hajnal, A., \& Rado, R. (1965). Partition relations for cardinal numbers. Acta Mathematica Academiae Scientiarum Hungarica, 16, 93-196.

Feferman, S., Friedman, H., Maddy, P., \& Steel, J. (2000). Does mathematics need new axioms? The Bulletin of Symbolic Logic, 6, 401-446.

Lolli, G. (1977). On Ramsey's theorem and the Axiom of Choice. Notre Dame Journal of Formal Logic, 18(4), 599-601.

Mathias, A. R. D. (1977). Happy families. Annals of Mathematical Logic, 12(1), 59-111.

Mycielski, J. \& Steinhaus, H. (1962). A mathematical axiom contradicting the axiom of choice. Bulletin de Académie Polonaise des Sciences. Série des Sciences Mathématiques, Astronomiques et Physiques, 10,1-3.

Paris, J. \& Harrington, L. A. (1977). A mathematical incompleteness in Peano Arithmetic. In J. Barwise (Ed.), Handbook of mathematical logic (pp. 1133-1142). Amsterdam: North-Holland.

Patey, L. \& Yokoyama, K. (2016). The proof-theoretic strength of Ramsey's theorem for pairs and two colors. ArXiv e-prints

Di Prisco, C. A. \& Henle, J. M. (1993). Partitions of products. Journal of Symbolic Logic, 58(3), 860-871.

Di Prisco, C. A., \& Henle, J. (1999). Partitions of the reals and choice. In X. Caicedo, \& C. H. Montenegro (Eds.), Selected Papers of the X Latin American Symposium on Mathematical Logic (pp. 13-23). Marcel Dekker, Inc.

Di Prisco, C. A. \& Todorcevic, S. (2003). Souslin partitions of products of finite sets. Advances in Mathematics, 176, 145-173.

Quine, W. V. O. (1969). Natural kinds. In J. Kim \& E. Sosa (Eds.), Ontological relativity and other essays (pp. 114-138). New York: Columbia University Press.

Ramsey, F. P. (1930). On a problem of formal logic. Proceedings of the London Mathematical Society, 30, 264-286.

Shelah, S. (1998). A polarized partition relation and failure of $\mathrm{GCH}$ at singular strong limit. Fundamenta Mathematicae, 155(2), 153-160.

Wittgenstein, L. (1998). Culture and value (P. Winch, Trans.). Basil Blackwell. Organized by G. $\mathrm{H}$. von Wright in collaboration with H. Nyman. 